\title{
Discovery of the Lensed Quasar System DES J0408-5354
}

H. Lin ${ }^{1}$, E. Buckley-Geer ${ }^{1}$, A. Agnello ${ }^{2}$, F. Ostrovski ${ }^{3,4}$, R. G. McMahon ${ }^{3,4}$, B. Nord ${ }^{1}$, N. Kuropatkin ${ }^{1}$, D. L. Tucker ${ }^{1}$, T. Treu $^{5}$, J. H. H. Chan ${ }^{6,7}$, S. H. Suyu ${ }^{7,6,8}$, H. T. Diehl ${ }^{1}$, T. Collett', M. S. S. Gill ${ }^{10}$, A. More ${ }^{11}$, A. Amara ${ }^{12}$, M. W. Auger ${ }^{3}$, F. Courbin $^{13}$, C. D. Fassnacht ${ }^{14}$, J. Frieman ${ }^{1,15}$, P. J. Marshall ${ }^{16}$, G. Meylan $^{13}$, C. E. Rusu ${ }^{17}$, T. M. C. Abbott ${ }^{18}$, F. B. Abdalla ${ }^{1920}$, S. Allam ${ }^{1}$, M. Banerji ${ }^{3,4}, K_{\text {Bechtol }}^{21}$, A. Benoit-Lévy ${ }^{22,19,23}$, E. Bertin ${ }^{22,23}$, D. Brooks ${ }^{19}$, D. L. Burke ${ }^{24,10}$, A. Carnero Rosell ${ }^{25,26}$, M. Carrasco Kind ${ }^{27,28}$, J. Carretero ${ }^{29}$, F. J. Castander ${ }^{30}$, M. Crocce ${ }^{30}$, C. B. D’Andrea ${ }^{31}$, L. N. da Costa ${ }^{25,26}$, S. Desai ${ }^{32}$, J. P. Dietrich ${ }^{33,34}$, T. F. Eifler ${ }^{35}$, D. A. Finley ${ }^{1}$, B. Flaugher ${ }^{1}$, P. Fosalba ${ }^{30}$, J. García-Bellido ${ }^{36}$, E. Gaztanaga ${ }^{30}$, D. W. Gerdes ${ }^{37,38}$, D. A. Goldstein ${ }^{39,40}$, D. Gruen ${ }^{24,10}$, R. A. Gruendl ${ }^{27,28}$, J. Gschwend ${ }^{25,26}$, G. Gutierrez ${ }^{1}$, K. Honscheid ${ }^{41,42}$, D. J. James ${ }^{43,18}$,

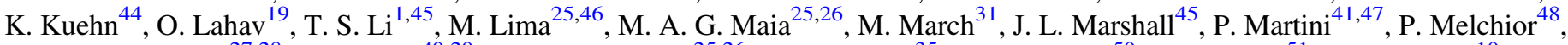
F. Menanteau ${ }^{27,28}$, R. Miquel ${ }^{49,29}$, R. L. C. Ogando ${ }^{25,26}$, A. A. Plazas ${ }^{35}$, A. K. Romer ${ }^{50}$, E. Sanchez ${ }^{51}$, R. Schindler ${ }^{10}$, M. Schubnell ${ }^{38}$, I. Sevilla-Noarbe ${ }^{51}$, M. Smith ${ }^{52}$, R. C. Smith ${ }^{18}$, F. Sobreira ${ }^{25,53}$, E. Suchyta ${ }^{54}$, M. E. C. Swanson ${ }^{28}$, G. Tarle $^{38}$, D. Thomas ${ }^{9}$, and A. R. Walker ${ }^{18}$

(DES Collaboration)

${ }^{1}$ Fermi National Accelerator Laboratory, P.O. Box 500, Batavia, IL 60510, USA

${ }^{2}$ European Southern Observatory, Karl-Schwarzschild-Strasse 2, D-85748 Garching bei München, Germany ${ }^{3}$ Institute of Astronomy, University of Cambridge, Madingley Road, Cambridge CB3 OHA, UK

${ }^{4}$ Kavli Institute for Cosmology, University of Cambridge, Madingley Road, Cambridge CB3 OHA, UK

${ }^{5}$ Department of Physics and Astronomy, University of California, Los Angeles, CA 90095, USA

${ }^{6}$ Institute of Astronomy and Astrophysics, Academia Sinica, P.O. Box 23-141, Taipei 10617, Taiwan

${ }^{7}$ Max-Planck-Institut fur Astrophysik, Karl-Schwarzschild-Str. 1, D-85741 Garching, Germany

${ }^{8}$ Physik-Department, Technische Universität München, James-Franck-Straße 1, D-85748 Garching, Germany

${ }^{9}$ Institute of Cosmology \& Gravitation, University of Portsmouth, Portsmouth PO1 3FX, UK ${ }^{10}$ SLAC National Accelerator Laboratory, Menlo Park, CA 94025, USA

${ }^{11}$ Kavli Institute for the Physics and Mathematics of the Universe (WPI), University of Tokyo Institutes for Advanced Study (UTIAS), University of Tokyo, Chiba, 277-8583, Japan

${ }^{12}$ Department of Physics, ETH Zurich, Wolfgang-Pauli-Strasse 16, CH-8093 Zurich, Switzerland

${ }^{13}$ Laboratoire d'Astrophysique, Ecole Polytechnique Fédérale de Lausanne (EPFL), Observatoire de Sauverny, CH-1290 Versoix, Switzerland

${ }^{14}$ Department of Physics, University of California Davis, 1 Shields Avenue, Davis, CA 95616, USA

${ }^{15}$ Kavli Institute for Cosmological Physics, University of Chicago, Chicago, IL 60637, USA

${ }^{16}$ Kavli Institute for Particle Astrophysics and Cosmology, Stanford University, 452 Lomita Mall, Stanford, CA 94305, USA

${ }^{17}$ Department of Physics, University of California, Davis, One Shields Avenue, Davis, CA 95616, USA

${ }^{18}$ Cerro Tololo Inter-American Observatory, National Optical Astronomy Observatory, Casilla 603, La Serena, Chile

${ }_{19}$ Department of Physics \& Astronomy, University College London, Gower Street, London WC1E 6BT, UK

${ }^{20}$ Department of Physics and Electronics, Rhodes University, P.O. Box 94, Grahamstown 6140, South Africa ${ }^{21}$ LSST, 933 North Cherry Avenue, Tucson, AZ 85721, USA

${ }^{22}$ CNRS, UMR 7095, Institut d'Astrophysique de Paris, F-75014, Paris, France

${ }^{23}$ Sorbonne Universités, UPMC Univ Paris 06, UMR 7095, Institut d'Astrophysique de Paris, F-75014 Paris, France

${ }^{24}$ Kavli Institute for Particle Astrophysics \& Cosmology, P.O. Box 2450, Stanford University, Stanford, CA 94305, USA

${ }^{25}$ Laboratório Interinstitucional de e-Astronomia-LIneA, Rua Gal. José Cristino 77, Rio de Janeiro, RJ-20921-400, Brazil

${ }^{26}$ Observatório Nacional, Rua Gal. José Cristino 77, Rio de Janeiro, RJ-20921-400, Brazil

${ }^{27}$ Department of Astronomy, University of Illinois, 1002 W. Green Street, Urbana, IL 61801, USA

${ }^{28}$ National Center for Supercomputing Applications, 1205 West Clark Street, Urbana, IL 61801, USA

${ }^{29}$ Institut de Física d'Altes Energies (IFAE), The Barcelona Institute of Science and Technology, Campus UAB, E-08193 Bellaterra, Barcelona, Spain

${ }^{30}$ Institut de Ciències de l'Espai, IEEC-CSIC, Campus UAB, Carrer de Can Magrans, s/n, E-08193 Bellaterra, Barcelona, Spain

${ }^{31}$ Department of Physics and Astronomy, University of Pennsylvania, Philadelphia, PA 19104, USA

${ }^{32}$ Department of Physics, IIT Hyderabad, Kandi, Telangana 502285, India

${ }^{33}$ Excellence Cluster Universe, Boltzmannstr. 2, D-85748 Garching, Germany

${ }^{34}$ Faculty of Physics, Ludwig-Maximilians-Universität, Scheinerstr. 1, D-81679 Munich, Germany

${ }^{35}$ Jet Propulsion Laboratory, California Institute of Technology, 4800 Oak Grove Drive, Pasadena, CA 91109, USA

${ }^{36}$ Instituto de Fisica Teorica UAM/CSIC, Universidad Autonoma de Madrid, E-28049 Madrid, Spain

37 Department of Astronomy, University of Michigan, Ann Arbor, MI 48109, USA

${ }^{39}$ Department of Astronomy, University of California, Berkeley, 501 Campbell Hall, Berkeley, CA 94720, USA

${ }^{40}$ Lawrence Berkeley National Laboratory, 1 Cyclotron Road, Berkeley, CA 94720, USA
${ }^{41}$ Center for Cosmology and Astro-Particle Physics, The Ohio State University, Columbus, OH 43210, USA

${ }^{42}$ Department of Physics, The Ohio State University, Columbus, OH 43210, USA

${ }^{43}$ Astronomy Department, University of Washington, Box 351580, Seattle, WA 98195, USA ${ }^{44}$ Australian Astronomical Observatory, North Ryde, NSW 2113, Australia

${ }^{45}$ George P. and Cynthia Woods Mitchell Institute for Fundamental Physics and Astronomy, and Department of Physics and Astronomy, Texas A\&M University, College Station, TX 77843, USA

${ }^{46}$ Departamento de Física Matemática, Instituto de Física, Universidade de São Paulo, CP 66318, CEP 05314-970, São Paulo, SP, Brazil

${ }^{47}$ Department of Astronomy, The Ohio State University, Columbus, OH 43210, USA

${ }^{48}$ Department of Astrophysical Sciences, Princeton University, Peyton Hall, Princeton, NJ 08544, USA

${ }^{49}$ Institució Catalana de Recerca i Estudis Avançats, E-08010 Barcelona, Spain

${ }^{50}$ Department of Physics and Astronomy, Pevensey Building, University of Sussex, Brighton BN1 9QH, UK

${ }^{51}$ Centro de Investigaciones Energéticas, Medioambientales y Tecnológicas (CIEMAT), Madrid, Spain

${ }^{52}$ School of Physics and Astronomy, University of Southampton, Southampton SO17 1BJ, UK

${ }^{53}$ Universidade Federal do ABC, Centro de Ciências Naturais e Humanas, Av. dos Estados, 5001, Santo André, SP, 09210-580, Brazil 
${ }^{54}$ Computer Science and Mathematics Division, Oak Ridge National Laboratory, Oak Ridge, TN 37831, USA
Received 2017 January 31; revised 2017 February 20; accepted 2017 February 20; published 2017 March 27

\begin{abstract}
We report the discovery and spectroscopic confirmation of the quad-like lensed quasar system DES J0408-5354 found in the Dark Energy Survey (DES) Year 1 (Y1) data. This system was discovered during a search for DES Y1 strong lensing systems using a method that identified candidates as red galaxies with multiple blue neighbors. DES J0408-5354 consists of a central red galaxy surrounded by three bright $(i<20)$ blue objects and a fourth red object. Subsequent spectroscopic observations using the Gemini South telescope confirmed that the three blue objects are indeed the lensed images of a quasar with redshift $z=2.375$, and that the central red object is an earlytype lensing galaxy with redshift $z=0.597$. DES J0408-5354 is the first quad lensed quasar system to be found in DES and begins to demonstrate the potential of DES to discover and dramatically increase the sample size of these very rare objects.
\end{abstract}

Key words: gravitational lensing: strong - quasars: general - surveys

\section{Introduction}

Strong gravitational lensing systems provide valuable tools for studying the properties and evolution of galaxies and quasars, for measuring the distribution of dark matter, and for constraining cosmological parameters. In particular, lensed quasar systems can provide information on, for example, intervening absorption line systems (e.g., Smette et al. 1995), properties of quasar host galaxies (e.g., Peng et al. 2006), dark matter substructure in the lens (e.g., Dalal \& Kochanek 2002), and the stellar content of the lensing galaxy (e.g., Schechter et al. 2014). Moreover, when combined with time delay measurements and careful lens modeling, lensed quasar systems can provide powerful cosmological constraints that are complementary to those of other techniques (e.g., Suyu et al. 2016; Treu \& Marshall 2016; Bonvin et al. 2017).

Previously, large samples of lensed quasars have been found by surveys such as the Cosmic Lens All-Sky Survey (Browne et al. 2003; Myers et al. 2003) in the radio and the Sloan Digital Sky Survey Quasar Lens Search (SQLS; Oguri et al. 2006; Inada et al. 2012) and the SDSS-III BOSS quasar lens survey (More et al. 2016) in the optical. The Dark Energy Survey (DES; Dark Energy Survey Collaboration 2005, 2016) is an ongoing imaging survey covering $5000 \mathrm{deg}^{2}$ of the Southern Galactic Cap in the grizY filters using the Dark Energy Camera (Flaugher et al. 2015), and it holds the promise of significantly increasing the numbers of lensed quasars. In particular, based on the forecasts of Oguri \& Marshall (2010), we expect to find in DES about 120 lensed quasar systems brighter than $i=21$ (magnitude limit applies to the fainter image for pairs and third brightest image for quadruple systems, or quads; see Figure 1 of Ostrovski et al. 2017). DES, specifically with the external collaboration project STRIDES ${ }^{55}$ (PI: T. Treu), aims to use the resulting large lensed quasar sample for the primary science goal of improving constraints on cosmological parameters. This lensed quasar sample is predicted to include about 20 of the very rare quad systems that will provide additional valuable information compared to pair systems for constraining lens models (specifically extra constraints from two more positions and two more time delays), in particular for cosmology purposes (e.g., Suyu et al. 2013) and substructure studies (e.g., Dalal \& Kochanek 2002).

To date, we have discovered and spectroscopically confirmed three lensed quasar pair systems (Agnello et al. 2015b;

\footnotetext{
55 http://strides.astro.ucla.edu/
}

Ostrovski et al. 2017) in DES. Here in this Letter we report the discovery and confirmation of the first lensed quasar quad (or quad-like) system, DES J0408-5354, in the DES. We first describe our lensed quasar search procedure, system discovery, and photometric data in Section 2. We then describe our spectroscopic observations and present our data in Section 3. We summarize and conclude in Section 4. Detailed photometry analysis and lens modeling for this system are presented in a modeling paper, Agnello et al. (2017), to which we will refer the reader where relevant below.

\section{Search and Discovery}

The system DES J0408-5354 was discovered during a systematic visual search for candidate strong lensing systems in the DES Year 1 (Y1) data (Diehl et al. 2014). A number of different search methods have been applied to the DES Y1 data, but the specific method involved in this case was a "bluenear-red" technique, where we first automatically identified candidate systems of red galaxies with multiple neighboring blue objects within some radius, and then used visual inspections of these systems to rate them and to select the best candidates for subsequent spectroscopic follow-up. This method has been used previously in the Sloan Digital Sky Survey (SDSS) data to search for bright strong lensing systems and then to confirm them spectroscopically with good success (e.g., Belokurov et al. 2009; Diehl et al. 2009).

Here, we started with a DES Y1 red galaxy sample selected using the redMaGiC technique (Rozo et al. 2016). To minimize stellar contamination of this sample, we rejected objects with SExtractor (Bertin \& Arnouts 1996) spread_model (Desai et al. 2012; Bouy et al. 2013) values $\leqslant 0.01$. We then identified our initial set of candidates as those redMaGiC galaxies with three or more blue objects within a radius $<10^{\prime \prime}$, where we defined a blue object as one with colors $-1<=g-r<1$ and $-1<=r-i<1$. We did not apply any star/galaxy separation cut to the blue objects, but did reject objects that are saturated in any of the $g, r, i$ filters using a SExtractor flags $<=3$ cut. We also applied a magnitude cut $r<22$ on the blue objects to keep the number of candidates manageable for the visual inspection step and to have relatively brighter candidates to ease follow-up spectroscopic redshift measurements.

Applying the above criteria resulted in a list of 6526 systems that one of us (HL) then inspected visually by examining their DES gri color composite images. The discovery image of DES 

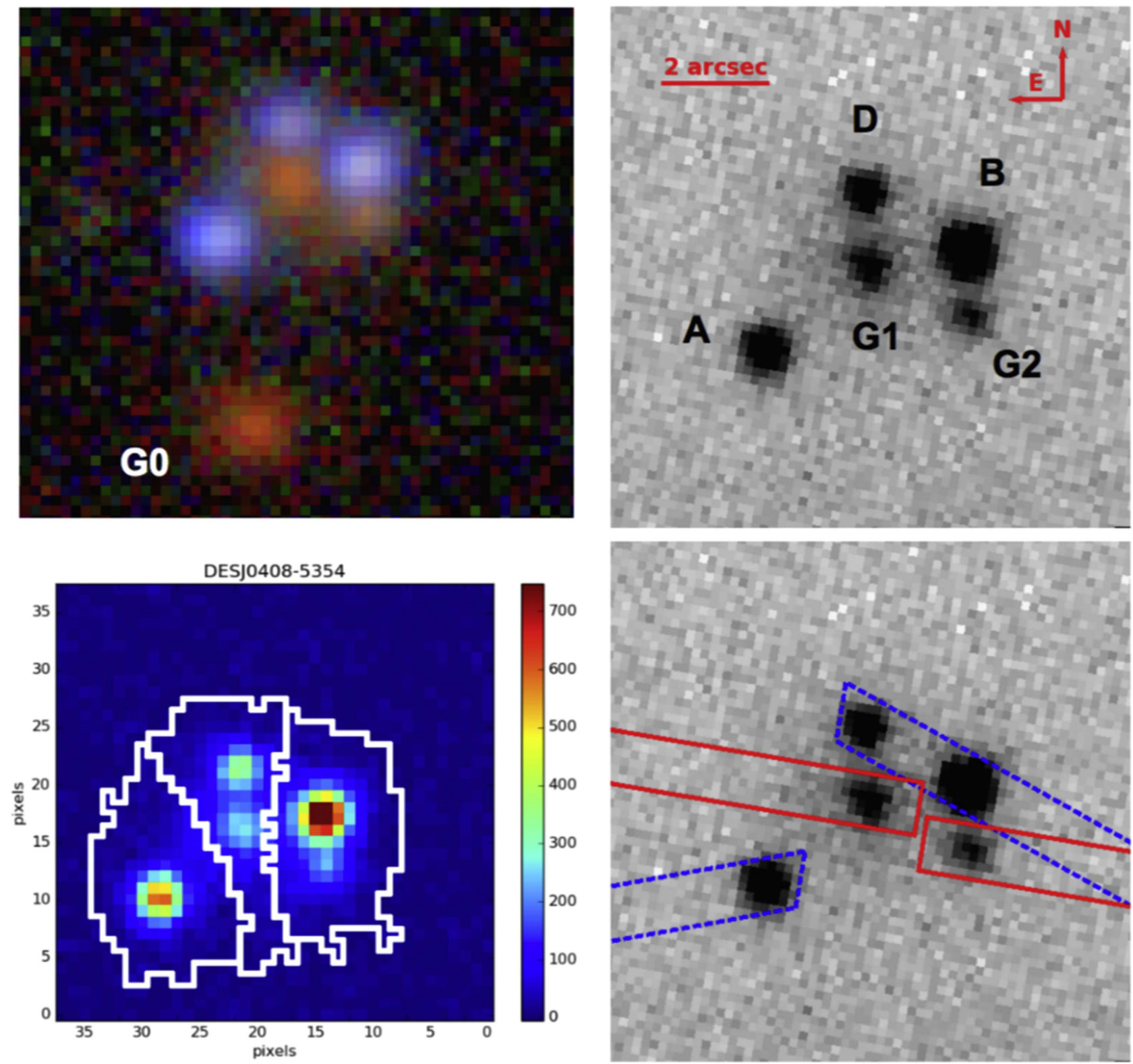

Figure 1. DES gri color composite discovery image (top left), SExtractor segmentation map plotted over the DES $i$-band coadded image (bottom left), Gemini $i$ band acquisition image (top right), and the spectroscopic slit layout (bottom right). The central red lensing galaxy is G1; the three blue lensed quasar images are A, B, and D; a fourth red image is G2; and the redMaGiC galaxy is G0. The 5 components (A, B, D, G1, G2) of the system are not fully separated in the DES catalog, with $\mathrm{D}+\mathrm{G} 1$ and $\mathrm{B}+\mathrm{G} 2$ remaining blended.

J0408-5354 is shown in Figure 1, where we see a central red galaxy (G1) surrounded by three blue objects (A, B, and D) and a fourth red object $(\mathrm{G} 2)$ (objects labeled as in Agnello et al. 2017). This system stood out as a potential quadruply lensed quasar system, except that the fourth putative lensed quasar image has a conspicuously redder color than those of the other three images. Regardless, the quad-like configuration of DES J0408-5354 made it a very good candidate for subsequent spectroscopy, described in the next section. We present a detailed lensing model for this system in Agnello et al. (2017), which concludes that the system is indeed a quad where the reddened image is a blend of the fourth lensed quasar image with a perturbing galaxy.

In the DES Y1 catalog, DES J0408-5354 is composed of three objects due to the fact that components D and G1 are blended and that B and G2 are also blended. As such, the catalog contains entries from $\mathrm{A}, \mathrm{D}+\mathrm{G} 1$, and $\mathrm{B}+\mathrm{G} 2$. The SExtractor segmentation map for the system is shown in Figure 1, overplotted on the DES $i$-band image of the system. We need to point out here that all three catalog objects actually meet our blue object color criteria given before (see Figure 2, leftmost panel), so that the redMaGiC galaxy for this system is not G1 but rather the red galaxy G0 marked in Figure 1. G0 is about 6 " away from $\mathrm{G1}$ and has a redMaGiC photometric redshift of $0.66 \pm 0.03$, close to G1's spectroscopic redshift of 0.597 (see Section 3). Therefore, in this case, our blue-near-red method found the system via another red galaxy likely associated with the lensing galaxy, rather than directly via the red lensing galaxy itself. The discovery was thus likely not coincidental, but was also not by the direct route as intended by the method. In similar DES Y1 blue-near-red and related searches, we also see other cases in galaxy group and cluster 

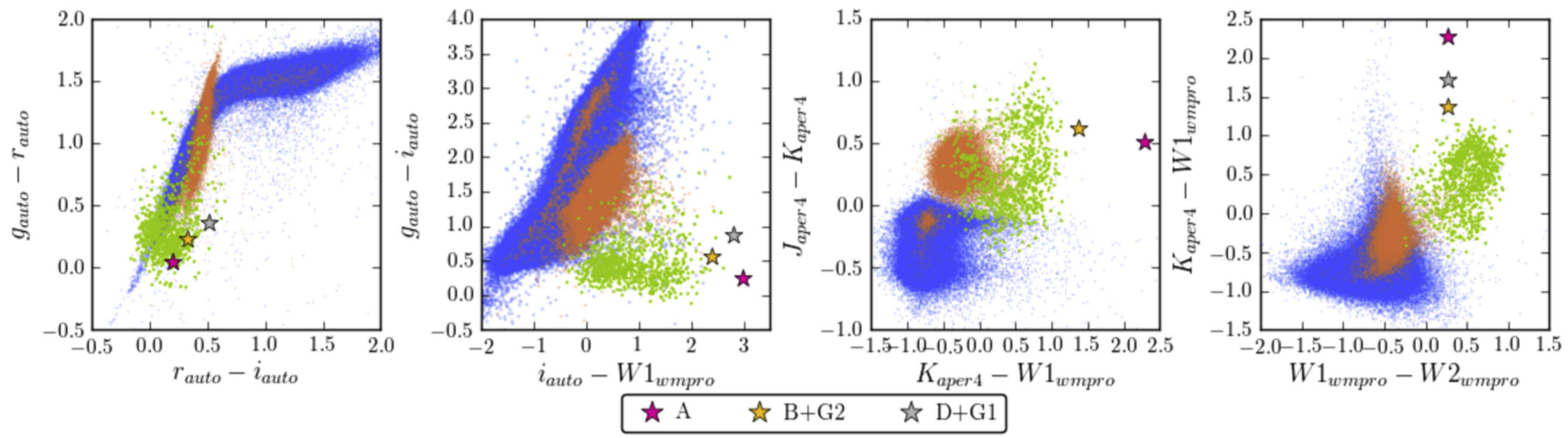

Figure 2. Color-color plots showing components A (pink stars), D $+\mathrm{G} 1$ (gray stars), and B+G2 (yellow stars) in different color spaces. We did not assign $W 1$ or $W 2$ fluxes to each individual component, so values show the sum of all flux in those bands. For comparison, the color loci of quasars (green), point sources (blue), and extended sources (orange) are populated by the objects present in the GMM training set.

Table 1

DES J0408-5354 Positions (J2000 Coordinates) and Photometry (AB Magnitudes)

\begin{tabular}{|c|c|c|c|c|c|c|c|c|c|}
\hline & A & $\mathrm{D}+\mathrm{G} 1$ & $\mathrm{~B}+\mathrm{G} 2$ & A & B & $\mathrm{D}$ & G1 & G2 & G0 \\
\hline R.A. & $4^{\mathrm{h}} 8^{\mathrm{m}} 21^{\mathrm{s}} \cdot 92$ & $4^{\mathrm{h}} 8^{\mathrm{m}} 21^{\mathrm{s}} .71$ & $4^{\mathrm{h}} 8^{\mathrm{m}} 21^{\mathrm{s}} .50$ & $4^{\mathrm{h}} 8^{\mathrm{m}} 21^{\mathrm{s}} \cdot 92$ & $4^{\mathrm{h}} 8^{\mathrm{m}} 21^{\mathrm{s}} \cdot 49$ & $4^{\mathrm{h}} 8^{\mathrm{m}} 21^{\mathrm{s}} .71$ & $4^{\mathrm{h}} 8^{\mathrm{m}} 21^{\mathrm{s}} \cdot 70$ & $4^{\mathrm{h}} 8^{\mathrm{m}} 21^{\mathrm{s}} .49$ & $4^{\mathrm{h}} 8^{\mathrm{m}} 21^{\mathrm{s}} .83$ \\
\hline Decl. & $-53^{\circ} 54^{\prime} 1^{\prime \prime} 0$ & $-53^{\circ} 53^{\prime} 58^{\prime \prime} 7$ & $-53^{\circ} 53^{\prime} 59^{\prime \prime} 2$ & $-53^{\circ} 54^{\prime} 1^{\prime \prime} 0$ & $-53^{\circ} 53^{\prime} 59^{\prime \prime} .2$ & $-53^{\circ} 53^{\prime} 58 !^{\prime \prime} 1$ & $-53^{\circ} 53^{\prime} 59^{\prime \prime} 6$ & $-53^{\circ} 54^{\prime} 0 ! \prime 3$ & $-53^{\circ} 54^{\prime} 5^{\prime \prime} 9$ \\
\hline$g$ & $19.99 \pm 0.01$ & $20.45 \pm 0.01$ & $19.74 \pm 0.01$ & $20.07 \pm 0.07$ & $19.98 \pm 0.07$ & $20.90 \pm 0.07$ & $22.18 \pm 0.20$ & $22.68 \pm 0.20$ & $23.46 \pm 0.19$ \\
\hline$r$ & $19.94 \pm 0.01$ & $20.08 \pm 0.01$ & $19.51 \pm 0.01$ & $20.16 \pm 0.07$ & $19.95 \pm 0.07$ & $20.94 \pm 0.10$ & $20.65 \pm 0.03$ & $21.98 \pm 0.15$ & $21.68 \pm 0.05$ \\
\hline$i$ & $19.75 \pm 0.01$ & $19.57 \pm 0.01$ & $19.18 \pm 0.01$ & $20.16 \pm 0.07$ & $19.74 \pm 0.10$ & $20.73 \pm 0.12$ & $19.77 \pm 0.04$ & $21.46 \pm 0.15$ & $20.37 \pm 0.03$ \\
\hline$z$ & $19.51 \pm 0.02$ & $19.13 \pm 0.02$ & $18.82 \pm 0.01$ & $19.96 \pm 0.10$ & $19.28 \pm 0.08$ & $20.42 \pm 0.10$ & $19.31 \pm 0.03$ & $20.91 \pm 0.12$ & $19.90 \pm 0.04$ \\
\hline$Y$ & $19.48 \pm 0.07$ & $19.09 \pm 0.05$ & $18.69 \pm 0.03$ & $20.04 \pm 0.10$ & $19.34 \pm 0.10$ & $20.77 \pm 0.13$ & $19.12 \pm 0.05$ & $20.56 \pm 0.16$ & $19.79 \pm 0.12$ \\
\hline$J$ & $19.58 \pm 0.06$ & $\ldots$ & $18.77 \pm 0.03$ & & & & & & \\
\hline$H$ & $19.62 \pm 0.08$ & $18.90 \pm 0.04$ & $18.65 \pm 0.03$ & & & & & & \\
\hline$K$ & $19.06 \pm 0.07$ & $18.51 \pm 0.04$ & $18.15 \pm 0.03$ & & & & & & \\
\hline \multicolumn{10}{|c|}{$\mathrm{A}+\mathrm{D}+\mathrm{G} 1+\mathrm{B}+\mathrm{G} 2$} \\
\hline$W 1$ & & $16.78 \pm 0.02$ & & & & & & & \\
\hline$W 2$ & & $16.51 \pm 0.02$ & & & & & & & \\
\hline
\end{tabular}

environments where the lensing system is being indirectly found this way (H. T. Diehl et al. 2017, in preparation).

The coordinates and photometry of the three DES catalog objects are given in Table 1. The DES SExtractor auto magnitudes of the three catalog objects are listed in Table 1, as are the near-infrared Kron magnitudes (Kron 1980) from the VISTA Hemisphere Survey (VHS; McMahon et al. 2013) and the mid-infrared magnitudes from the Wide-field Infrared Survey Explorer (WISE; Wright et al. 2010). For the nearinfrared catalog, magnitudes were obtained by cross-matching the DES and VHS catalogs using a 1"!5 search radius. Component $\mathrm{D}+\mathrm{G} 1$ does not have a $J$-band magnitude value due to blending with $\mathrm{B}+\mathrm{G} 2$ in this band. For the mid-infrared magnitudes, the DES and WISE catalogs were cross-matched using a 4!" 0 search radius. All components of the system are blended into a single WISE source, so that the $W 1$ and $W 2$ magnitudes displayed in the table apply to $\mathrm{A}+\mathrm{D}+\mathrm{G} 1+\mathrm{B}+\mathrm{G} 2$ as a whole.

We present in Agnello et al. (2017) a detailed analysis that provides the deblended positions and griz $Y$ magnitudes of all five components A, B, D, G1, and G2 in this system. For completeness we also provide this information in Table 1, as well as include the DES catalog position and photometry for galaxy G0.
After DES J0408-5354 was first announced to the STRIDES Collaboration as a lensed quasar candidate from the blue-near-red search, a number of other search methods within STRIDES were examined and also seen to identify the system as a candidate. These other search methods are described in more detail in Agnello et al. (2017). We provide here a brief summary: (1) the Gaussian Mixture Model (GMM) method of Ostrovski et al. (2017), which uses supervised machine learning in a five-dimensional optical plus infrared color space and identified DES J0408-5354 as a candidate pair (D+G1 not found separately in $J$ band due to blending noted above); (2) CHITAH (Chan et al. 2015), which uses pixel-based automatic recognition on griz $Y$ cutout images and identified the system as a candidate pair (not flagged as a quad because the fourth image G2 is too red); and (3) the Artificial Neural Network method of Agnello et al. (2015a, 2015b), which uses griz and W1W2 magnitudes and identified DES J0408-5354 as a candidate extended quasar. Figure 2 shows how the system was flagged by the GMM method as a candidate, due to the quasar-like colors of its components. We note that none of the search methods mentioned in this Letter selected DES J0408-5354 as a quad candidate, due to blending issues and/or reddended colors. The unusual color and configuration of this system and potential others like it argue for incorporating more flexibility into our search techniques (Agnello et al. 2017). 

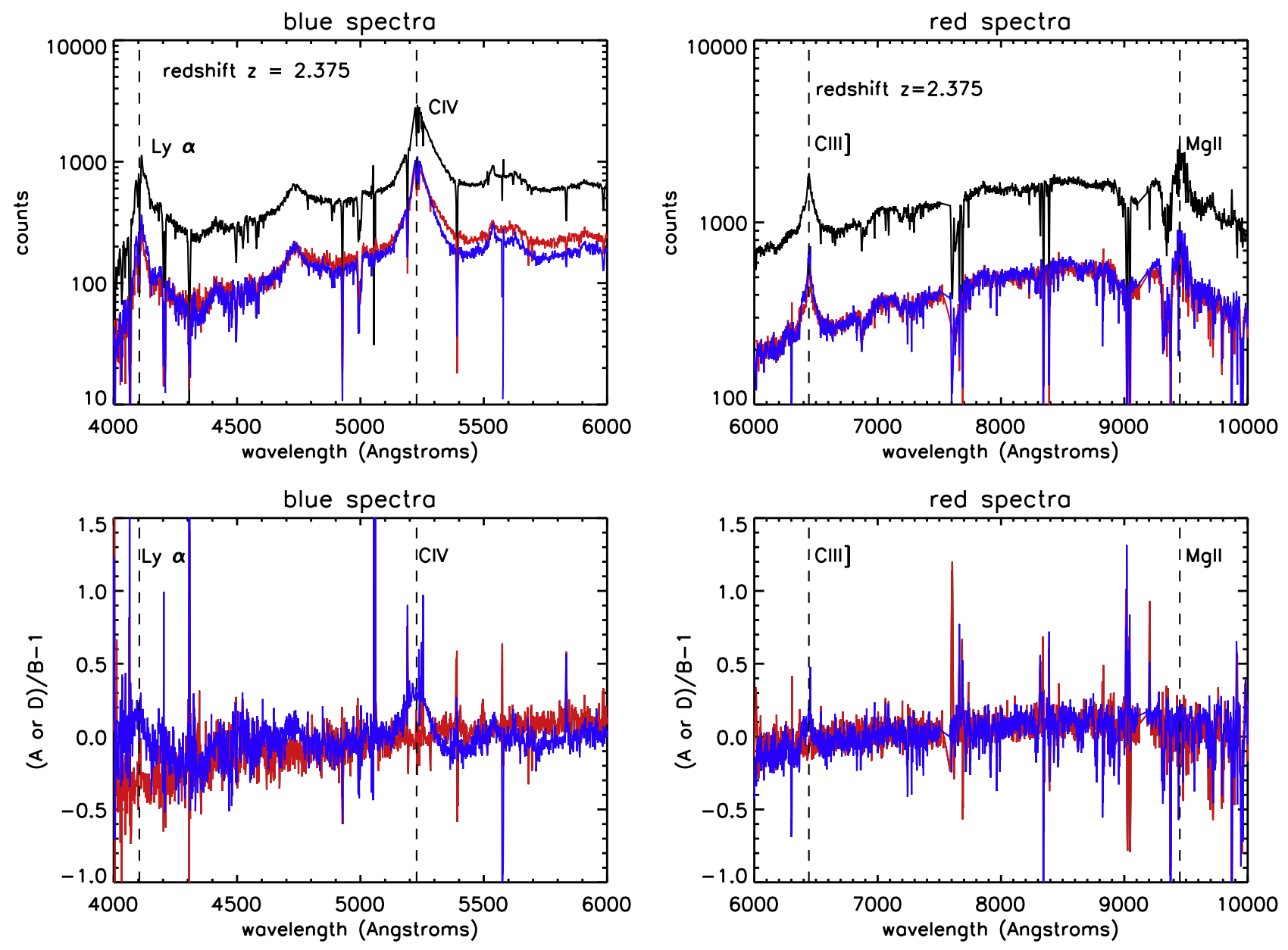

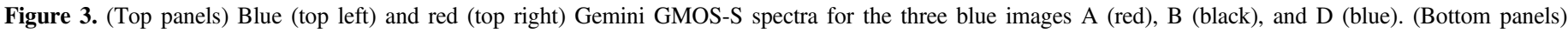

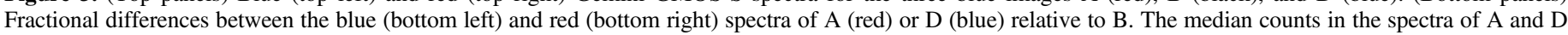
have been rescaled to match those of $\mathrm{B}$, over the respective blue and red wavelength ranges shown.

\section{Spectroscopic Observations}

Spectroscopic observations of DES J0408-5354 were carried out using the Gemini Multi-Object Spectrograph (GMOS-S) on the Gemini South telescope, as part of a larger Gemini Large and Long Program (LLP; PI: E. Buckley-Geer; program IDs GS-2015B-LP-5, GS-2016A-LP-5) of spectroscopic follow-up for DES strong lensing systems and for DES photometric redshift (photo-z) calibrations. GMOS-S was used in multiobject spectroscopy (MOS) mode on 2015 December 9 UT to take spectra of the three blue objects A, B, and D. The $i$-band GMOS-S acquisition image is shown in Figure 1 (top right). A single slit mask was used, with one slit for A and a second slit for B and D together; see blue slits in Figure 1 (bottom right). (Another 38 slits were assigned to unrelated DES photo- $z$ calibration targets.) Two sets of spectra were taken: blue data using the $\mathrm{B} 600$ grating (dispersion $\approx 1.0 \AA \mathrm{pixel}^{-1}$ ) and red data using the $\mathrm{R} 400$ grating (dispersion $\approx 1.5 \AA \mathrm{pixel}^{-1}$ ). We used $4 \times 900 \mathrm{~s}$ exposures for cosmic ray rejection and processed the data using the IRAF Gemini reduction package. The seeing was $0 . " 8$, measured from spectra of mask-alignment stars in the science data.

From the extracted (but not flux calibrated) 1D spectra shown in Figure 3 (top panels), we clearly see that all three blue images $\mathrm{A}, \mathrm{B}$, and $\mathrm{D}$ show strong quasar emission lines at the same redshift, specifically Ly $\alpha 1216 \AA$ and C IV $1549 \AA$ in the blue spectra, and C III] $1909 \AA$ and Mg II $2800 \AA$ in the red spectra. From the C III] line, which shows the cleanest, most symmetric line profile among these four broad emission features, we use the emsao task in the IRAF external package rvsao (Kurtz \& Mink 1998) to report a redshift $z=2.375$ for the quasar.

To compare the spectra of the three blue images in more detail, we also show in Figure 3 (bottom panels) the fractional differences between the blue and red spectra of images A and D relative to those of image $\mathrm{B}$, which has the spectra with the most counts. We first rescale the spectra of $\mathrm{A}$ and $\mathrm{D}$ so that they each have the same median counts as those of B over the respective blue and red wavelength ranges plotted. The results in Figure 3 show generally good agreement among the spectra, especially for the red spectra. For the blue spectra, we do see an overall slope for the spectrum of A relative to that of $\mathrm{B}$; we attribute this slope to extinction differences between the lines of sight to these two images, and/or to differences in atmospheric differential refraction between the two differently oriented slits (Figure 1) used to observe images A and B. In addition, we also see conspicuous differences between the spectra of D and B at the locations of the strong Ly $\alpha$ and C IV emission lines, and to a lesser extent at the C III] line. We attribute these differences to the effects of microlensing by stars in the lensing galaxy, manifested as flux ratio differences, in pairs of lensed quasar images, for the continuum versus the 

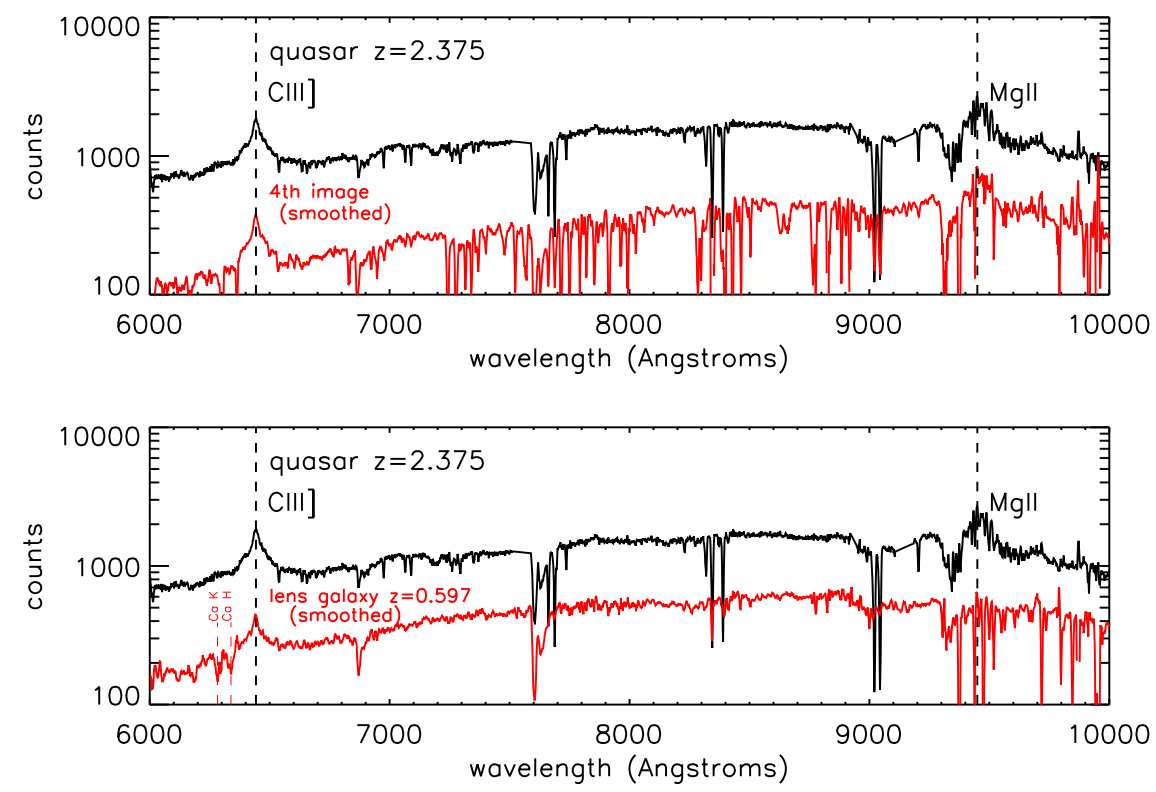

Figure 4. Red Gemini GMOS-S spectra of the fourth image G2 (top panel, red) and of the central lensing galaxy G1 (bottom panel, red). In both panels, the black spectrum is that of image $B$.

emission line regions in the spectra (e.g., Motta et al. 2012). Microlensing may also cause the spectral slope differences mentioned above.

Subsequent to these observations that confirmed the three blue images as having the same quasar redshift, we designed a second MOS slit mask targeting the central lensing galaxy G1 and the fourth image G2, each allocated to a single slit. The slits for G1 and G2 are shown in red in Figure 1 (bottom right). Red R400 observations were obtained in the Gemini South semester 2016A observing queue, on 2016 April 9, under 1"'1 seeing conditions and using the same observing setup as above. Blue B600 observations were also put into the queue, but no data were obtained before the system set in 2016A. We show the R400 spectra in Figure 4, where we see that G2 shows broad C III] and $\mathrm{Mg}$ II emission at the same redshift as found for the three blue images, while $\mathrm{G} 1$ shows $\mathrm{Ca} \mathrm{H}+\mathrm{K}$ absorption lines, indicating that G1 is an early-type lensing galaxy with redshift $z=0.597$.

However, we also see that the G1 spectrum unexpectedly shows the same C III] emission line as in the lensed quasar spectra, leading us to suspect there is contaminating light from the neighboring lensed quasar image $\mathrm{D}$, which is only about 1" 3 away (cf. the 1 "! 1 seeing). Likewise, object B, the brightest quasar image, is only about 1 !" 1 away from the substantially fainter object $\mathrm{G} 2$, suggesting that the $\mathrm{C}$ III] line seen in the G2 spectrum is also contamination from object $\mathrm{B}$. Using the image modeling code GALFIT (Peng et al. 2010), we simulate image B as a Moffat profile with an FWHM of 1". 1 and find that about $12 \%$ of its light falls within the $1^{\prime \prime}$-wide slit and $2^{\prime \prime}$-long spectral extraction aperture used for G2. Though seemingly small, this $12 \%$ fraction actually amounts to about $80 \%$ of the $r$-band light from G2 itself, given that $r(\mathrm{~B})=19.95$ and $r(\mathrm{G} 2)=21.98$ from Table 1 (Agnello et al. 2017). (We use $r$ band as the C III] line falls within that filter.) This estimated contamination is thus substantial and unfortunately we are unable to correct for it as image B lies off the slit for G2 (Figure 1, bottom right) and we do not have a concurrent spectrum of B. We thus cannot rule out contamination from B as the source of the $\mathrm{C}$ III] line seen in the $\mathrm{G} 2$ spectrum (likewise for D and G1) and will need future observations to verify whether G2 indeed shows quasar emission features or not.

\section{Summary and Conclusions}

DES J0408-5354 was identified as a candidate lensed quasar quad system from the DES first-year data. Subsequent followup spectroscopy confirmed the three bright blue objects in this system to be the images of a quasar at redshift $z=2.375$, lensed by an early-type red galaxy with redshift $z=0.597$. Another reddened object in the system, possibly a blend of a perturbing galaxy and the fourth lensed quasar image, was also observed spectroscopically, though without conclusive results.

Our modeling paper, Agnello et al. (2017), presents a detailed model of the system, from which we expect the longest time delay in the system to be about 80 days, making DES J0408-5354 well suited (Treu \& Marshall 2016) for time delay measurements, which we are undertaking via a monitoring campaign within the STRIDES Collaboration. The lensing model also constrains the mass of the possible perturbing galaxy and thus provides information about substructure in the lensing mass distribution. Further spectroscopic follow-up and high-resolution imaging data should provide more needed details about the main lensing galaxy and its environment. We thus expect this quad system to be particularly useful for the application of time delay cosmography (e.g., Treu \& Marshall 2016) and substructure studies (e.g., Dalal \& Kochanek 2002). DES J0408-5354 heralds a much larger sample of some 20 of these very rare and valuable quad lensed quasar systems anticipated to be discovered by the DES.

Funding for the DES Projects has been provided by the U.S. Department of Energy, the U.S. National Science Foundation, the Ministry of Science and Education of Spain, the Science and Technology Facilities Council of the United Kingdom, the Higher Education Funding Council for England, the National Center for Supercomputing Applications at the University of Illinois at Urbana-Champaign, the Kavli Institute of Cosmological Physics at the University of Chicago, the Center for 
Cosmology and Astro-Particle Physics at the Ohio State University, the Mitchell Institute for Fundamental Physics and Astronomy at Texas A\&M University, Financiadora de Estudos e Projetos, Fundação Carlos Chagas Filho de Amparo à Pesquisa do Estado do Rio de Janeiro, Conselho Nacional de Desenvolvimento Científico e Tecnológico and the Ministério da Ciência, Tecnologia e Inovação, the Deutsche Forschungsgemeinschaft and the Collaborating Institutions in the Dark Energy Survey.

The Collaborating Institutions are Argonne National Laboratory, the University of California at Santa Cruz, the University of Cambridge, Centro de Investigaciones Energéticas, Medioambientales y Tecnológicas-Madrid, the University of Chicago, University College London, the DES-Brazil Consortium, the University of Edinburgh, the Eidgenössische Technische Hochschule (ETH) Zürich, Fermi National Accelerator Laboratory, the University of Illinois at Urbana-Champaign, the Institut de Ciències de l'Espai (IEEC/CSIC), the Institut de Física d'Altes Energies, Lawrence Berkeley National Laboratory, the Ludwig-Maximilians Universität München and the associated Excellence Cluster Universe, the University of Michigan, the National Optical Astronomy Observatory, the University of Nottingham, The Ohio State University, the University of Pennsylvania, the University of Portsmouth, SLAC National Accelerator Laboratory, Stanford University, the University of Sussex, Texas A\&M University, and the OzDES Membership Consortium.

The DES data management system is supported by the National Science Foundation under grant number AST1138766. The DES participants from Spanish institutions are partially supported by MINECO under grants AYA201239559, ESP2013-48274, FPA2013-47986, and Centro de Excelencia Severo Ochoa SEV-2012-0234. Research leading to these results has received funding from the European Research Council under the European Union's Seventh Framework Programme FP7/2007-2013) including ERC grant agreements 240672, 291329, and 306478 .

Based on observations obtained at the Gemini Observatory (acquired through the Gemini Observatory Archive and processed using the Gemini IRAF package), which is operated by the Association of Universities for Research in Astronomy, Inc., under a cooperative agreement with the NSF on behalf of the Gemini partnership: the National Science Foundation (United States), the National Research Council (Canada),
CONICYT (Chile), Ministerio de Ciencia, Tecnología e Innovación Productiva (Argentina), and Ministério da Ciência, Tecnologia e Inovação (Brazil).

The analysis presented here is based on observations obtained as part of the VISTA Hemisphere Survey, ESO Programme, 179.A-2010 (PI: McMahon).

\section{References}

Agnello, A., Kelly, B. C., Treu, T., \& Marshall, P. J. 2015a, MNRAS, 448,1446

Agnello, A., Lin, H., Buckley-Geer, E., et al. 2017, MNRAS, submitted (arXiv:1702.00406)

Agnello, A., Treu, T., Ostrovski, F., et al. 2015b, MNRAS, 454, 1260

Belokurov, V., Evans, N. W., Hewett, P. C., et al. 2009, MNRAS, 392, 104

Bertin, E., \& Arnouts, S. 1996, A\&AS, 117, 393

Bonvin, V., Courbin, F., Suyu, S. H., et al. 2017, MNRAS, 465, 4914

Bouy, H., Bertin, E., Moraux, E., et al. 2013, A\&A, 554, 101

Browne, I. W. A., Wilkinson, P. N., Jackson, N. J. F., et al. 2003, MNRAS, 341,13

Chan, J. H. H., Suyu, S. H., Chiueh, T., et al. 2015, ApJ, 807, 138

Dalal, N., \& Kochanek, C. S. 2002, ApJ, 572, 25

Dark Energy Survey Collaboration 2005, arXiv:astro-ph/0510346

Dark Energy Survey Collaboration 2016, MNRAS, 460, 1270

Desai, S., Armstrong, R., Mohr, J. J., et al. 2012, ApJ, 757, 83

Diehl, H. T., Abbott, T. M. C., Annis, J., et al. 2014, Proc. SPIE, 9149, 91490V

Diehl, H. T., Allam, S. S., Annis, J., et al. 2009, ApJ, 707, 686

Flaugher, B., Diehl, H. T., Honscheid, K., et al. 2015, AJ, 150, 150

Inada, N., Oguri, M., Shin, M.-S., et al. 2012, AJ, 143, 119

Kron, R. G. 1980, ApJS, 43, 305

Kurtz, M. J., \& Mink, D. J. 1998, PASP, 110, 934

McMahon, R. G., Banerji, M., Gonzalez, E., et al. (VHS Collaboration) 2013 , Msngr, 154, 35

More, A., Oguri, M., Kayo, I., et al. 2016, MNRAS, 456, 1595

Motta, V., Mediavilla, E., Falco, E., \& Muñoz, J. A. 2012, ApJ, 755, 82

Myers, S. T., Jackson, N. J., Browne, I. W. A., et al. 2003, MNRAS, 341, 1

Oguri, M., Inada, N., Pindor, B., et al. 2006, AJ, 132, 999

Oguri, M., \& Marshall, P. J. 2010, MNRAS, 405, 2579

Ostrovski, F., McMahon, R. G., Connolly, A. J., et al. 2017, MNRAS, 465,4325

Peng, C. Y., Ho, L. C., Impey, C. D., \& Rix, H.-W. 2010, AJ, 139, 2097

Peng, C. Y., Impey, C. D., Rix, H.-W., et al. 2006, ApJ, 649, 616

Rozo, E., Rykoff, E. S., Abate, A., et al. 2016, MNRAS, 461, 1431

Schechter, P. L., Pooley, D., Blackburne, J. A, \& Wambsganss, J. 2014, ApJ, 793, 96

Smette, A., Robertson, J. G., Shaver, P. A., et al. 1995, A\&AS, 113, 199

Suyu, S. H., Auger, M. W., Hilbert, S., et al. 2013, ApJ, 766, 70

Suyu, S. H., Bonvin, V., Courbin, F., et al. 2016, MNRAS, submitted (arXiv:1607.00017)

Treu, T., \& Marshall, P. J. 2016, A\&ARv, 24, 11

Wright, E. L., Eisenhardt, P. R. M., Mainzer, A. K., et al. 2010, AJ, 140, 1868 